\title{
Inhibition of ERK1/2 down-regulates the Hippo/YAP signaling pathway in human NSCLC cells
}

\author{
Bin You ${ }^{1,2, *}$, Yi-Lin Yang ${ }^{1, *}$, Zhidong $X \mathbf{u}^{1}$, Yuyuan Dai ${ }^{1}$, Shu Liu ${ }^{1}$, Jian-Hua Mao ${ }^{3}$, \\ Osamu Tetsu ${ }^{4}$, Hui Li ${ }^{2}$, David M. Jablons', Liang You ${ }^{1}$ \\ ${ }^{1}$ Department of Surgery, Helen Diller Family Comprehensive Cancer Center, University of California, San Francisco, CA, USA \\ ${ }^{2}$ Department of Thoracic Surgery, Beijing Chao-Yang Hospital, Affiliated with Capital University of Medical Science, Beijing, \\ People's Republic of China \\ ${ }^{3}$ Life Sciences Division, Lawrence Berkeley National Laboratory, University of California, Berkeley, CA, USA \\ ${ }^{4}$ Department of Otolaryngology - Head and Neck Surgery, University of California, San Francisco, CA, USA \\ *These authors have contributed equally to this work
}

Correspondence to:

Liang You, e-mail: liang.you@ucsfmedctr.org

Keywords: non-small cell lung cancer, extracellular signal regulated kinases, Hippo pathway, yes-associated protein, inhibition

Received: June 17, 2014

Accepted: December 20, 2014

Published: January 23, 2015

\section{ABSTRACT}

Alterations of the EGFR/ERK and Hippo/YAP pathway have been found in nonsmall cell lung cancer (NSCLC). Herein, we show that ERK1 and ERK2 have an effect on the Hippo/YAP pathway in human NSCLC cells. Firstly, inhibition of ERK1/2 by siRNA or small-molecular inhibitors decreased the YAP protein level, the reporter activity of the Hippo pathway, and the mRNA levels of the Hippo downstream genes, CTGF, Gli2, and BIRC5. Secondly, degradation of YAP protein was accelerated after ERK1/2 depletion in NSCLC cell lines, in which YAP mRNA level was not decreased. Thirdly, forced over-expression of the ERK2 gene rescued the YAP protein level and Hippo reporter activity after siRNA knockdown targeting 3'UTR of the ERK2 gene in NSCLC cells. Fourthly, depletion of ERK1/2 reduced the migration and invasion of NSCLC cells. Combined depletion of ERK1/ 2 had a greater effect on cell migration than depletion of either one separately. Finally, the MEK1/2 inhibitor Trametinib decreased YAP protein level and transcriptional activity of the Hippo pathway in NSCLC cell lines. Our results suggest that ERK1/ 2 inhibition participates in reducing YAP protein level, which in turn down-regulates expression of the downstream genes of the Hippo pathway to suppress migration and invasion of NSCLC cells.

\section{INTRODUCTION}

Non-small cell lung cancer (NSCLC), a common malignancy, has a known association with mitogen-activated protein kinase (MAPK) signal transduction pathways [1-3]. Extracellular signal regulated kinases (ERK1/2) are important components of the MAPK signal pathway, which is mediated by Epidermal Growth Factor Receptor (EGFR). ERK $1 / 2$ proteins, after activation through the Ras-RafMEK kinase cascade, can translocate into the nucleus and activate various effectors, including transcriptional factors, kinases, and phosphatases, to promote cell differentiation and proliferation [4]. Amplification of genes that encode ERK and overexpression of ERK have been found in human NSCLC $[5,6]$. In clinical and pre-clinical studies, disrupting the MAPK/ERK pathway in cancer cells through inhibiting Raf or MEK often induced negative feedback or paradoxical activation of ERK and resulted in drug resistance [7-9]. Direct inhibition of ERK1/2 is becoming a new strategy in cancer treatment [10-12].

Another cancer pathway identified in NSCLC is the Hippo (also known as the Salvador-Warts-Hippo) pathway [13-15]. In normal cells, Yes-associated protein (YAP), one of the major mediators, is negatively regulated by upstream components of the Hippo pathway [16, 17]. The Hippo/YAP pathway has shown a correlation with stem cell renewal and differentiation, a crucial step of oncogenic transformation [18]. YAP plays a key role in regulating organ size and cancer development $[19,20]$, and as a growth promoter, YAP is found at elevated levels in many 
human cancers [21, 22]. Moreover, YAP up-regulation was identified in NSCLC patient tissues [13, 22].

Hippo is an evolutionarily conserved pathway in cells, and just a few oncogenic mutations have been discovered in human cancers to date [23]. Understanding what causes alteration of the Hippo pathway in cancer cells could lead to new therapeutic strategies. On the other hand, as a central transducer of many biological signals, ERK1/2 mediates a series of molecular alterations and has complex crosstalk with other signaling pathways [24-26]. We sought to investigate whether ERK1/2 expression is associated with Hippo pathway activity by analyzing the effects of ERK1/2 inhibition on the Hippo pathway activity in human NSCLC cells. We analyzed the transcriptional activity of Hippo downstream genes and YAP protein expression after ERK1/2 knockdown by using small interfering RNA (siRNA) or small-molecular inhibitors. We report for the first time that ERK1/2 inhibition contributes to downregulation of the Hippo pathway in human NSCLC cells and suppressed migration and invasion of the cell.

\section{RESULTS}

\section{Inhibition of ERK1/2 down-regulates YAP protein expression}

To investigate whether inhibition of ERK1/2 influences YAP, we examined the protein level of YAP after ERK1/2 knockdown. The efficiency of ERK inhibition after 48 hours of siRNA treatment in NSCLC H1975 and H2170 cells was assessed by western blotting (Figure 1A). We found that YAP protein level decreased after ERK1/2 knockdown by siRNA (Figure 1B). To verify the suppression of YAP protein by ERK inhibition, we analyzed YAP protein level in the NSCLC cells after treatment with small-molecular inhibitors. We used two ERK inhibitors: CAY10561 (N-[1-(3-chloro4-fluorophenyl)-2-hydroxyethyl]-4-[4-(3-chlorophenyl)1-Hpyrazol-3-yl]-1H-pyrrole-2-carboxamide), a highly selective inhibitor of ERK2 [27], and FR180204 (5(2-Phenyl-pyrazolo [1, 5-a] pyridin-3-yl)-1H-pyrazolo [3, 4-c] pyridazin-3-ylamine), a selective inhibitor of ERK1/2 [28]. The results showed that YAP expression decreased in a dose-dependent manner in both cell lines treated with either CAY10561 or FR180204, in contrast to what occurred after control treatment with DMSO (Figure 1C). These findings suggest that ERK1/2 inhibition decreases the protein level of YAP in NSCLC cells.

\section{ERK1/2 Inhibition does not decrease the mRNA level of YAP}

We next tested whether ERK1/2 inhibition decreases the mRNA level of YAP in NSCLC cells. The mRNA level of YAP after ERK1/2 siRNA treatment in H1975 and
H2170 cells was analyzed by using semi-quantitative realtime PCR (RT-PCR). We found that YAP mRNA levels in the cells treated with ERK1/2 siRNA did not differ significantly from those treated with non-targeting control siRNA (Figure 2A; Suppl. Table S1). These results suggest that ERK1/2 inhibition down-regulates YAP at the protein level rather than at the mRNA level.

\section{Depletion of ERK1/2 promotes YAP degradation}

To investigate whether the decrease of YAP protein level mediated by ERK1/2 inhibition is through degradation, we examined the half-life of YAP protein in H1975 and H2170 cells after ERK inhibition. After ERK1/2 inhibition by siRNA, cells were treated with protein inhibitor cycloheximide and YAP protein level was analyzed in the cells at $0,1,2,4,6$ and 8 hours by western blotting (Figure 2B). In H1975 cells, the half-life of YAP protein is about 6 hours after treatment of cycloheximide and non-targeting siRNA control. After ERK1/2 inhibition by siRNA, YAP protein reached a low level at 2 hours after cycloheximide treatment, suggesting that the halflife of YAP after ERK1/2 depletion is about 2 hours in H1975 cells. In H2170 cells treating with non-targeting siRNA, the YAP protein level showed only minor decrease within 8 hours. After ERK1/2 depletion, the YAP protein level decreased dramatically at 1 hour after cycloheximide treatment, suggesting a half-life of less than 1 hour in H2170 cells. These results showed that ERK1/2 depletion decreased the half-life of YAP in both NSCLC cell lines, suggesting ERK1/2 depletion promotes YAP degradation.

\section{ERK1/2 inhibition down-regulates hippo reporter activity and downstream gene expression}

As YAP is a central mediator of the Hippo pathway, we next investigated whether ERK1/2 suppression affects the reporter activity of the Hippo pathway in NSCLC cells. GTIIC reporter activity of Hippo pathway was analyzed in $\mathrm{H} 1975$ and $\mathrm{H} 2170$ cells after ERK inhibition by siRNA (Figure 3A). In H1975 cells, the reporter activity decreased by $16.5 \%$ after ERK1 silencing, $21.4 \%$ after ERK2 silencing, and 26.9\% after ERK1/2 silencing. In $\mathrm{H} 2170$ cells, the reporter activity decreased by $34.5 \%$ after ERK1 silencing, 9.8\% after ERK2 silencing, and $48.8 \%$ after ERK $1 / 2$ silencing. Our results show that ERK $1 / 2$ inhibition by siRNA significantly decreased GTIIC reporter activity of the Hippo pathway in both H1975 and H2170 cells $(P<0.05)$ compared to that of their respective control cells treated with non-targeting siRNA. Moreover, the mRNA level of the Hippo pathway downstream genes CTGF, Gli2, and BIRC5 analyzed in these cells was consistently down-regulated after ERK1/2 depletion (Figure 3B, Suppl. Table S2). Furthermore, Hippo reporter activity and Hippo downstream gene 
A

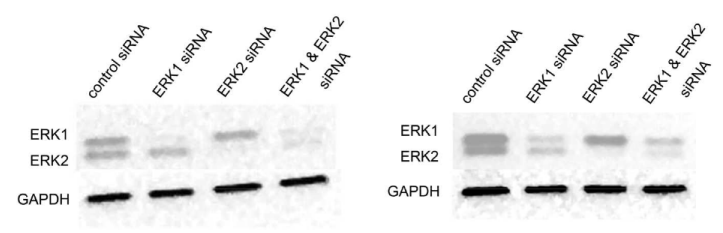

B
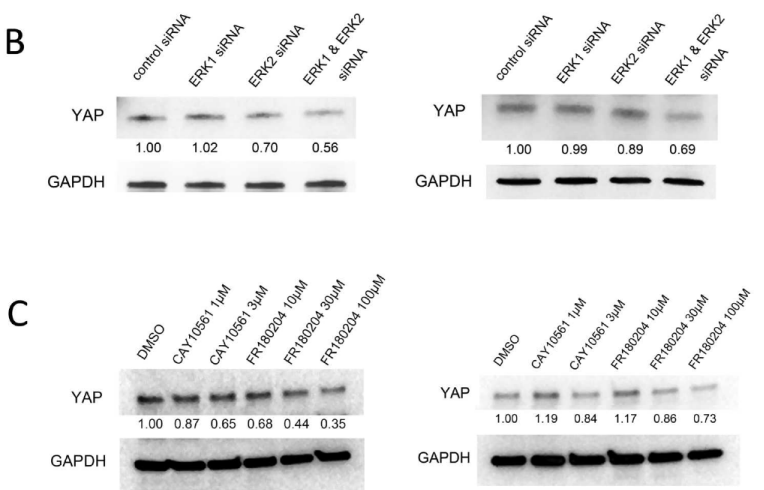

Figure 1: Western blotting analysis of YAP protein level after ERK1/2 inhibition in NSCLC cells. (A) Decreased ERK1 and ERK2 protein level after siRNA treatment in H1975 and H2170 cells. (B) Decreased YAP protein level after ERK1/2 inhibition by siRNAs $(100 \mathrm{nM})$ in H1975 and H2170 cells. (C) Dose-dependent decrease of YAP protein level in cells treated with ERK inhibitors.

A
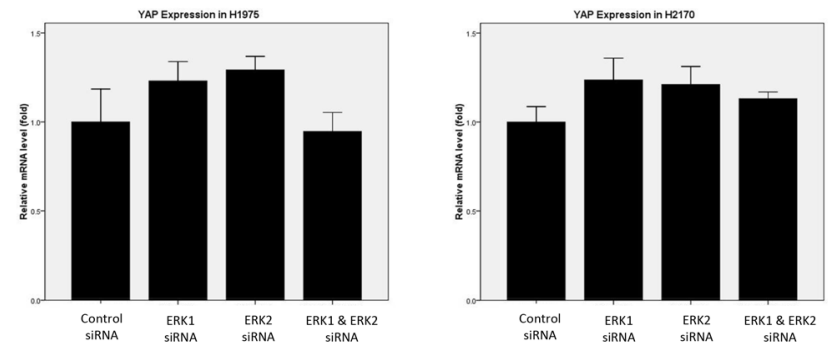

B
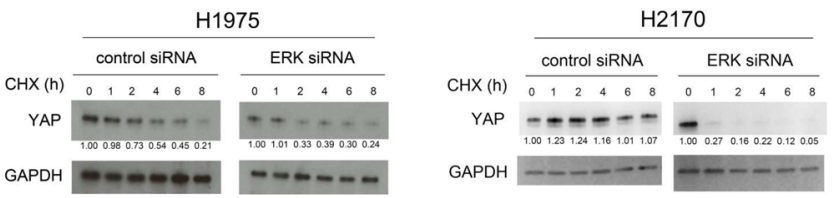

Figure 2: Analysis of YAP expression after ERK1/2 inhibition in NSCLC cells. (A) YAP mRNA level in H1975 and H2170 cells after ERK inhibition by siRNA was measured using RT-PCR. (B) Degradation of YAP protein was analyzed in H1975 and H2170 cells after ERK1/2 inhibition by siRNA.

transcription decreased more after depletion of both ERK1 and ERK2 than after depletion of ERK1 or ERK2 alone.

We further examined the effect of ERK inhibition on Hippo pathway activities using the small-molecular ERK2 inhibitor CAY10561 and the ERK1/2 inhibitor FR180204. After treatment with either inhibitor, Hippo reporter activity decreased in a dose-dependent manner in both H1975 and H2170 cells, as compared to the DMSO control $(P<0.05)$ (Figure 4A). Quantitative RTPCR analysis also showed a dose-dependent decrease of CTGF, Gli2 and BIRC5 transcription in both cell lines
$(P<0.05)$ (Figure 4B, Suppl. Table S3). Together, these results suggest that ERK1/2 inhibition down-regulates the reporter activity and downstream gene transcription of the Hippo pathway in NSCLC cells.

\section{Forced over-expression of the ERK2 gene rescues hippo/YAP expression during ERK2 depletion}

To verify that YAP protein expression can be regulated by ERK expression, we analyzed YAP protein level after 
A
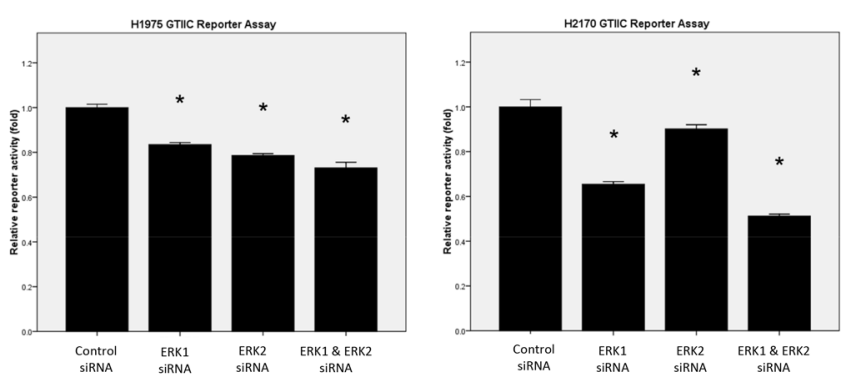

B
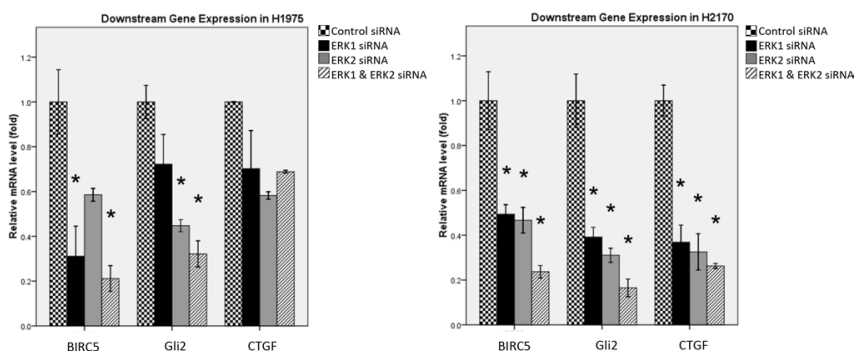

Figure 3: Analysis of Hippo pathway activity after ERK1/2 inhibition by siRNA in NSCLC cells. (A) GTIIC reporter activity of Hippo pathway after ERK1/2 inhibition by siRNA was analyzed in H1975 and H2170 cells $(* P<0.05$, one-way ANOVA and Scheffe multiple comparisons). (B) Decreased expression of BIRC5, Gli2, and CTGF, the downstream genes of Hippo pathway, after ERK1/2 inhibition by siRNA in H1975 and H2170 cells ( ${ }^{*} P<0.05$, One-way ANOVA and Scheffe multiple comparisons).

A

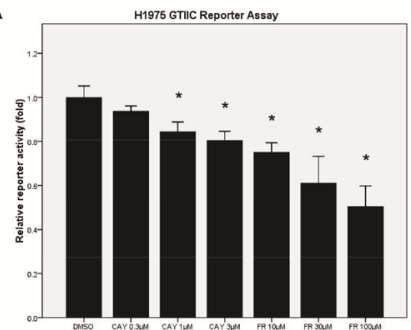

B

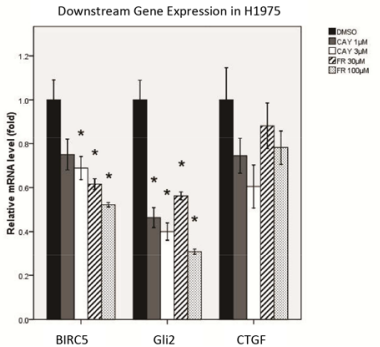

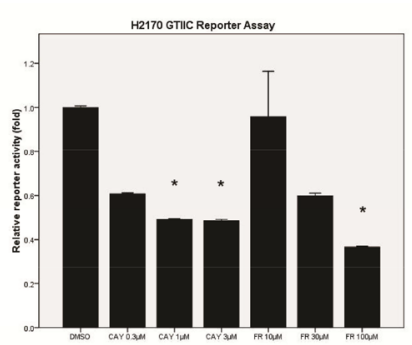

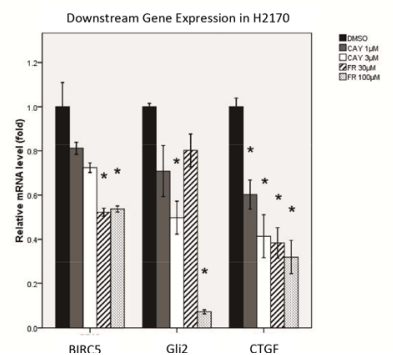

Figure 4: Analysis of Hippo pathway activity after ERK1/2 inhibition by small molecule inhibitors in NSCLC cells. (A) A dose-dependent decrease in GTIIC reporter activity of the Hippo pathway after ERK inhibition by ERK inhibitors (CAY10561 or FR180204) in H1975 and H2170 cells ( ${ }^{*} P<0.05$, One-way ANOVA and Scheffe multiple comparisons). (B) A dose-dependent decrease in mRNA level of BIRC5, CTGF, and Gli2, the downstream genes of Hippo pathway, after ERK inhibition by ERK inhibitors (CAY10561 and FR180204) in H1975 and H2170 cells ( ${ }^{*} P<0.05$, One-way ANOVA, Scheffe multiple comparisons).

ERK2 inhibition and/or forced over-expression of the ERK2 gene in NSCLC cell line A549. For this, we used the ERK2 siRNA, which targeted the 3'UTR end of the ERK2 gene. We found that YAP protein level decreased after ERK2 depletion in A549 cells (Figure 5A), results that were similar to what we found after ERK inhibition using a pooled ERK2 siRNA. After forced overexpression of the ERK2 gene, YAP protein level was $50 \%$ increase compared to that in the cells treated with ERK2 3'UTR siRNA only (Figure 5B). After 3'UTR siRNA treatment, Hippo reporter activity was significantly reduced by $62.6 \%$, compared to that in the cells treated with control non-targeting siRNA $(P<0.05)$, and Hippo reporter activity was rescued by more than $30 \%$ after forced overexpression of the ERK2 gene in cells $(P<0.05)$. Together, these results suggest that Hippo/YAP expression is regulated by ERK expression in NSCLC cells. 


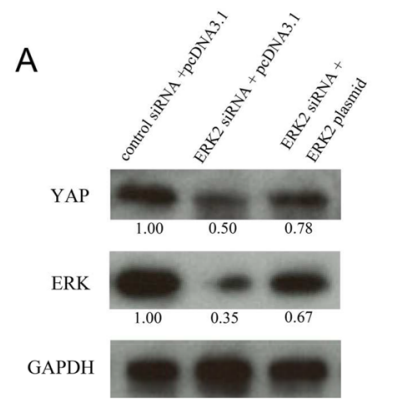

B

C

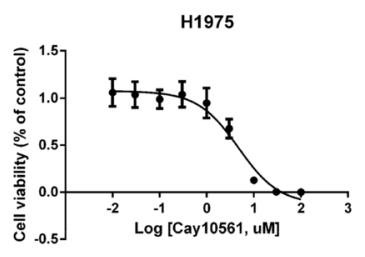

$\mathrm{D}$

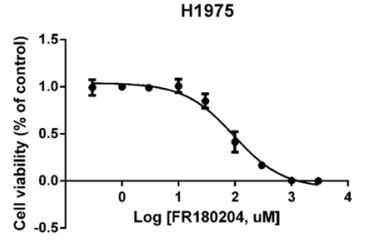

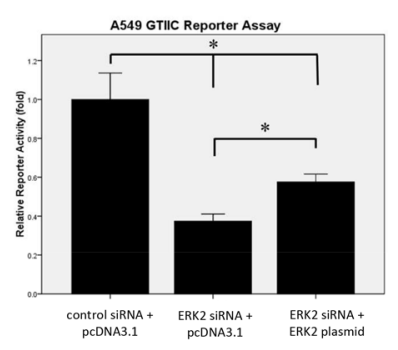

H2170

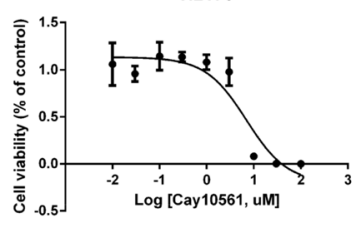

$\mathrm{H} 2170$

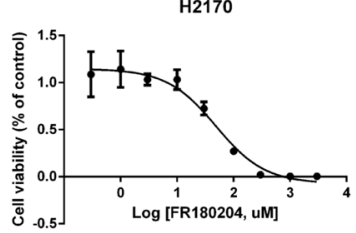

Figure 5: Expression of YAP/Hippo pathway and cell viability analysis after ERK inhibition in NSCLC cells. (A) Western blotting analysis of YAP, ERK, and GAPDH after ERK2 silencing by siRNA and/or forced over-expression of the ERK2 gene in A549 cells. (B) GTIIC reporter activity of the Hippo pathway after ERK2 silencing by siRNA and/or forced over-expression ERK2 gene in A549 cells $(* P<0.05$. One-way ANOVA and Scheffe multiple comparisons). (C) Cell viability analysis in H1975 and H2170 cells after ERK inhibitor CAY 10561 treatment. (D) Cell viability analysis in H1975 and H2170 cells after ERK inhibitor FR180204 treatment.

\section{ERK inhibitors suppress viability of NSCLC cells}

We next tested the effects of ERK inhibitors on the viability of NSCLC cells. H1975 and H2170 cells were treated with ERK inhibitors CA10561 and FR180204 at different doses for 48 hours. Cell viability was assayed and IC50 of each cell line was calculated based on the dose-response curves (Figure 5C, 5D). IC50 of CAY 10561 was $4.74 \mu \mathrm{M}$ in $\mathrm{H} 1975$ cells and $7.01 \mu \mathrm{M}$ in $\mathrm{H} 2170$ cells. IC50 of FR180204 in was $95.36 \mu \mathrm{M}$ in H1975 cells and $49.0 \mu \mathrm{M}$ in $\mathrm{H} 2170$ cells. These results show that ERK inhibition suppressed cell viability in a dose-dependent manner in both NSCLC cell lines.

\section{ERK1/2 inhibition restrains migration and invasion of NSCLC cells}

To assess the effect of ERK1/2 inhibition on the migration ability of NSCLC cells, we carried out a wound-healing assay using H1975 and H2170 cells. Cells transfected with ERK1/2 siRNA or YAP siRNA for 48 hours were scratched with a $200 \mu \mathrm{l}$ pipette tip, and the rate of wound closure was observed for 18 hours, when cells in the control group were proximally confluent. In both cell lines, wound closure rates were significantly decreased after ERK1/2 depletion compared to that in the control group (Figure 6A, 6B; $P<0.05$ ). Depletion of YAP yielded observations similar to those after depletion of both ERK1 and ERK2, suggesting that ERK1/2 inhibition restrains the migratory ability of the NSCLC tumor cells possibly through YAP down-regulation. Moreover, depletion of both ERK1 and ERK2 resulted in more significant inhibition of tumor cell migration than depletion of either alone $(P<0.05)$. This greater effect of ERK $1 / 2$ combined is consistent with our findings in the mRNA expression and reporter assay of Hippo pathway.

To test the effect of ERK inhibition on the invasion ability of NSCLC cells, a transwell assay was performed using H1975 cells after siRNA treatment for 48 hours. The number of the cells that invaded the lower side of the membrane decreased significantly 20 hours after ERK2 siRNA or YAP siRNA treatment, compared to that in the control group (Figure 6C, 6D; $P<0.05$ ). Similarly, the invasive ability of NSCLC cells was decreased when either ERK2 or YAP was depleted, suggesting that ERK2 inhibition suppresses the invasive ability of the NSCLC tumor cells possibly through the Hippo/YAP pathway. 


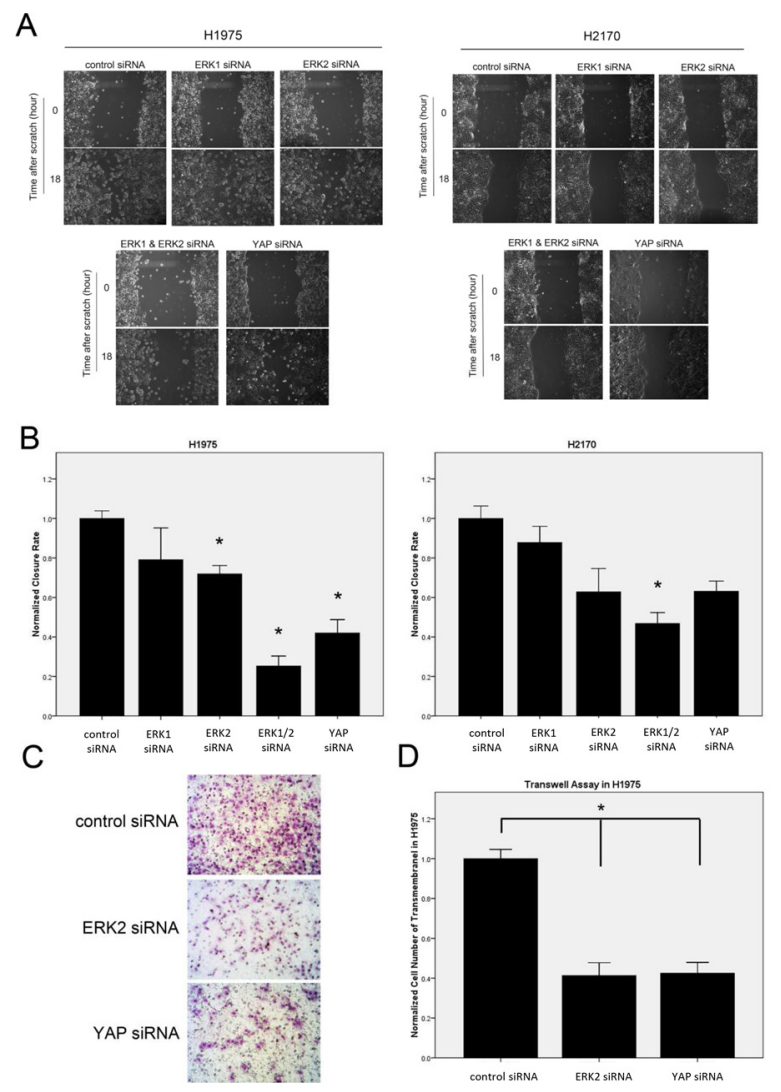

Figure 6: Analysis of cell migration and invasion abilities after ERK inhibition in NSCLC cells. (A) Decrease in cell migration ability after ERK or YAP inhibition by siRNA in H1975 and H2170 cells. (B) Quantitative analysis of migration assay result ( ${ }^{*} P<0.05$, one-way ANOVA, Scheffe multiple comparisons). (C) Decrease in cell invasion ability in H1975 cells after ERK2 or YAP silencing by siRNA. Images were taken under a $20 \times$ objective lens. (D) Quantitative analysis of the number of cells that invaded the lower side of the membrane in each experimental group $(* P<0.05$, One-way ANOVA and Scheffe multiple comparisons).

\section{MEK1/2 inhibition causes down-regulation of the hippo/YAP pathway}

To investigate whether inhibiting MEK, an upstream regulator of ERK signaling, affects Hippo pathway activity, we analyzed YAP expression in NSCLC A549 cells treated with the MEK1/2 inhibitor Trametinib (N- (3- (3- cyclopropyl-5-(2-fluoro-4-iodophenylamino)-6, 8-dimethyl-2, 4, 7-trioxo-3, 4, 6 ,7-tetrahydropyrido [4,3d] pyrimidin-1(2H)-yl) phenyl) acetamide, GSK1120212 [29] or the ERK inhibitor FR180204. We found that YAP protein level decreased after treatment with either inhibitor, in contrast with what occurred in cells treated with DMSO (Figure 7A). The mRNA level of YAP in A549 cells after Trametinib treatment was analyzed using RT-PCR, and there was no significant difference in YAP mRNA level in the cells with Trametinib or DMSO treatment (Figure 7B, Suppl. Table S4). Together, these results suggest that MEK inhibition decreased YAP protein level, but has only a minimal effect on YAP transcription in the cells. Furthermore, we examined the reporter activity and downstream gene expression of the Hippo pathway in NSCLC cells after MEK inhibition. GTIIC reporter activity of the Hippo pathway was decreased in A549 and H2170 cells after treatment with the MEK inhibitor Trametinib, compared to that of their respective control cells (Figure $7 \mathrm{C}, P<0.05$ ). Similarly, the mRNA levels of Hippo downstream genes, Gli2 and BIRC5, were consistently down-regulated by MEK inhibition in A549 cells (Figure 7D, Suppl. Table S5, $P<0.05$ ). The effect of MEK inhibition was similar to that of ERK inhibition on Hippo/YAP pathway activity. Our results suggest that MEK inhibition mediated YAP down-regulation at the protein level and thereby suppresses the reporter activity and downstream gene expression of the Hippo pathway in NSCLC cell lines.

\section{DISCUSSION}

The results of our study suggest that ERK1 and ERK2 have previously unrecognized effects on the Hippo pathway in human NSCLC cells. Several lines of evidence support this relationship between ERK1/2 and the Hippo/ YAP pathway. First, in NSCLC cell lines, ERK1/2 inhibition by siRNA or small-molecular inhibitors downregulates the protein level of YAP, and in turn suppresses transcriptional activity of the Hippo pathway. Additionally, these effects of ERK inhibition can be rescued by forced 
A

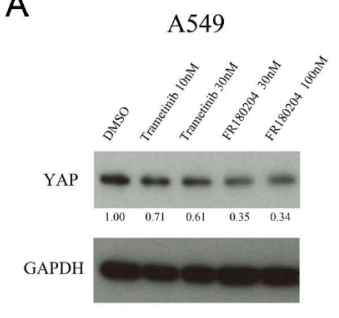

C
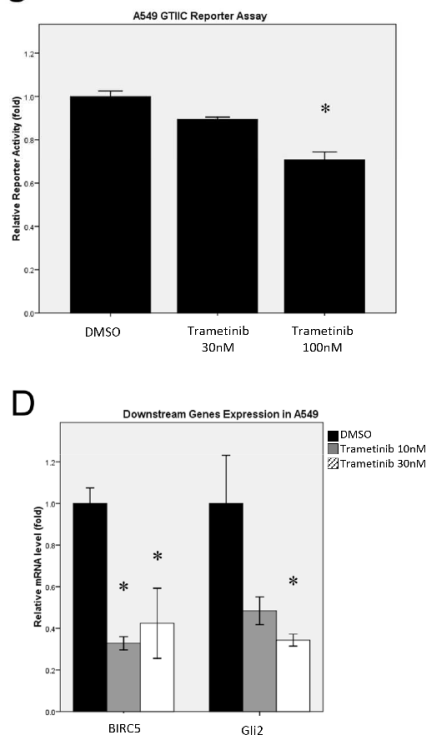

B
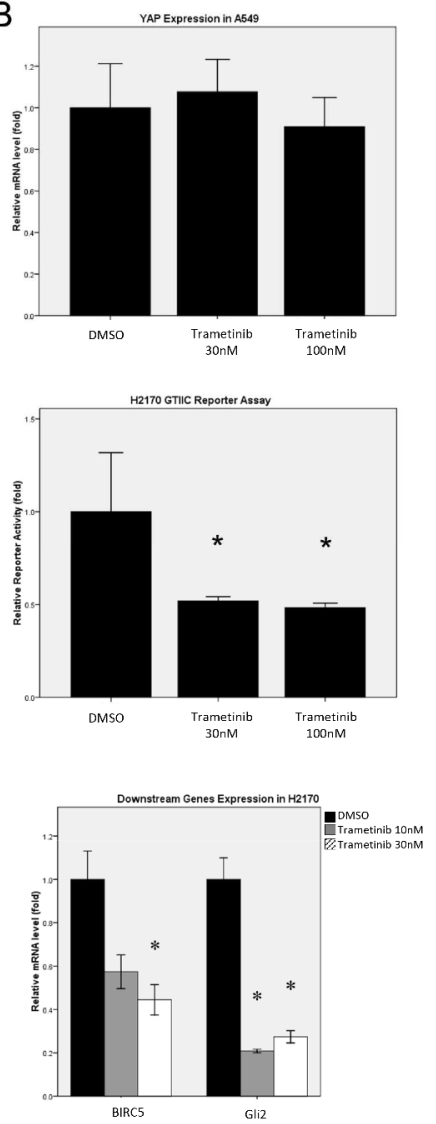

Figure 7: Analysis of Hippo/YAP pathway activity after MEK1/2 inhibition in NSCLC cells. (A) Western blotting analysis of YAP expression in A549 cells treated with MEK1/2 inhibitor Trametinib or ERK inhibitor FR180204. (B) The mRNA level of YAP in A549 cells treated with MEK1/2 inhibitor Trametinib was measured using RT-PCR. (C) A dose-dependent decrease in GTIIC reporter activity of the Hippo pathway was analyzed in A549 and $\mathrm{H} 2170$ cells treated with MEK1/2 inhibitor Trametinib $(* P<0.05$, One-way ANOVA and Scheffe multiple comparisons). (D) Decreased mRNA levels of BIRC5 and Gli2, the downstream genes of the Hippo pathway, in A549 and H2170 cells treated with MEK1/2 inhibitor Trametinib $(* P<0.05$, One-way ANOVA, Scheffe multiple comparisons).

over-expression of ERK2 in the cells. Second, ERK1/2 inhibition reduces the viability, migration, and invasion of NSCLC cells. Third, ERK1/2 knockdown decreased YAP expression at the protein level, but not the mRNA level, and the results of a protein degradation assay indicated that ERK1/2 inhibition down-regulates YAP protein level, at least partially, through promoting its degradation.

The correlation between ERK and the Hippo pathway, which have similar effects on cell properties and oncogenesis, has been studied. Mitogen-activated protein kinase 1 (MEK1) was recently reported to be closely correlated with YAP in human liver cancer cells, but ERK was deemed irrelevant to the Hippo/YAP pathway [30]. We analyzed the association of ERK and YAP expression in a lung cancer tissue array using immunohistochemistry. Our results show that YAP and ERK expression were significantly associated in 78 lung adenocarcinoma tumors (Suppl. Figure S1A-B; $P<0.05$ ). In addition, the results of our cell experiments suggest that ERK1/2 inhibition may negatively regulate the Hippo/YAP pathway in human NSCLC cells. Moreover, we performed a YAP rescue experiment after ERK2 knockdown by a 3'UTR
siRNA. The result shows that the viability of NSCLC cells was rescued by forced overexpression of the YAP gene (Suppl. Figure S2; $P<0.05$ ), suggesting that the suppressive effect of ERK inhibition on cellular biological behavior may be partly through down-regulating activity of the Hippo/YAP pathway. As the Hippo pathway is known to be a regulator of cancer stem cells [18, 31-33] and is involved in cell adhesion and migration, which are closely associated with cancer progression and metastasis [34-36], our results suggest that ERK $1 / 2$ should be a therapeutic target in this process.

Regulation of ERK expression is important in cancer cells [37, 38], and inhibitors of MEK, a kinase that activates ERK, have been studied in clinical trials. Our study shows that the MEK inhibitor Trametinib downregulated the Hippo/YAP pathway in a way that was similar to what occurred after ERK inhibition. However, inhibiting upstream components of the MAPK pathway, such as Raf or MEK, often induces negative feedback or paradoxical activation of ERK and results in drug resistance [7-9]. These findings, together with our results, suggest that the ERK inhibitor should be further evaluated 
for its therapeutic potential $[10,11,39]$. ERK inhibitors have already entered clinical trials for cancer treatment, for example, MK-8353/SCH900353, a clinical grade analogue of SCH772984, BVD-523, and RG7842 [40].

Targeting the Hippo pathway is a new direction for cancer drug development [41]. In human NSCLC, the Hippo pathway and some of its downstream genes, such as CTGF, Gli2 and BIRC5, are associated with the occurrence and development of the disease [42-45]. The results of our study show that in NSCLC cells, the expression of these Hippo downstream genes can be reduced by ERK1/2 inhibition and also suggest that the stability of YAP protein could be reduced by ERK1/2 inhibition. Therefore, blocking the Hippo pathway by ERK1/2 inhibition may be a therapeutic strategy in cancer treatment.

The mechanism by which ERK regulates YAP stability is not clear. In the Hippo pathway, YAP is negatively regulated by a core cassette consisted of LATS1, LATS2, MST1, and MST2 kinases [17, 23, 46]. In human NSCLC, LATS1 expression has been shown to be associated with YAP expression [47], and MST1 overexpression enhances YAP phosphorylation at the Ser127 site in human NSCLC cells [48]. Moreover, the Hippo pathway can be negatively regulated by epidermal growth factor (EGF) signaling [49], in which ERK functions as one of the vital effectors (Suppl. Figure S3). EGF signaling has been shown to dissociate the Hippo core complex, resulting in LATS inactivation, YAP dephosphorylation at Ser127, YAP nuclear accumulation, and CTGF transcriptional activation [50]. We detected nuclear staining of YAP in human NSCLC samples using immunohistochemistry (Suppl. Figure S1C), suggesting that EGF signaling is activated and regulates the Hippo pathway through inhibiting Hippo kinase(s) in the tumor cells. Other studies seeking to understand the regulation of Hippo pathway have shown that MAPK can regulate Ajuba family LIM domain protein (Jub), Wilms tumor protein 1-interacting protein (WTIP), or KIBRA, which are all regulators of the Hippo pathway [51, 52]. Raf-1 was also shown to regulate MST2 and the Hippo pathway [53]. Taken together, the results from our study and others lead us to hypothesize that ERK regulates YAP stability through the Hippo pathway in NSCLC cells. Further studies are warranted to test this hypothesis and to understand how MAPK regulates the Hippo pathway in human NSCLC.

In our study, the migration of cancer cells was inhibited more after ERK2 silencing than after ERK1 silencing, and depletion of both together showed a greater effect on Hippo pathway activity and cell migration than either of them did separately. These results suggest that inhibition of both ERK1 and ERK2 may be involved in regulating Hippo pathway activities. To date, research about ERK inhibitors has mainly focused on ERK2 [54], but collective inhibition of ERK1 and ERK2 may offer a better approach to therapy for human NSCLC.
In summary, we report that ERK1/2 inhibition down-regulates the protein level of YAP via promoting its degradation, and thereby reduces expression of the downstream genes of the Hippo pathway. This regulation of YAP may impair migratory and invasive activity of tumor cells in human NSCLC. Our results provide interesting insights about crosstalk between the two cell signaling pathways.

\section{METHODS}

\section{Cell culture}

Human NSCLC cell lines H1975, H2170 and A549 were obtained from American Type Culture Collections (Manassas, VA). Cell lines were maintained in RPMI1640 supplemented with $10 \%$ heat-inactivated fetal bovine serum, penicillin $(100 \mathrm{mg} / \mathrm{ml})$ and streptomycin $(100 \mathrm{mg} /$ $\mathrm{ml}$ ), and were cultured at $37^{\circ} \mathrm{C}$ in a humid incubator with $5 \% \mathrm{CO}_{2}$.

\section{Small molecules, siRNA, plasmid DNA, and antibodies}

The SMARTPool siRNA targeting p42 MAPK (ERK2), p44 MAPK (ERK1), and YAP were purchased from Thermo Scientific Dharmacon (Pittsburgh, PA). Nontargeting siRNA was used as control (Thermo Scientific Dharmacon, Pittsburgh, PA). Additional ERK2 siRNA (Hss163792) targeting the 3'UTR end of ERK2 gene purchased from Life Technologies (Grand Island, NY) was used in the rescue studies. The ERK2 inhibitor CAY10561 (CAS 933786-58-4) was obtained from Cayman Chemical Company (Ann Arbor, Michigan), the ERK1/2 inhibitor FR180204 (3706) from Tocris Bioscience (San Diego, CA), and the MEK inhibitor Trametinib from Selleckchem (Houston, TX). The pUSE ERK2 plasmid DNA used to over-express the ERK2 gene in the cells was purchased from Millipore. The pBaby-YAP plasmid DNA used to over-express the YAP gene in the cells was purchased from Addgene. Antibodies for ERK and YAP used in this study were purchased from Cell Signaling, Inc. (Danvers, MA). The HRP-linked secondary antibodies were from GE Healthcare Bio-Sciences Corp (Piscataway, NJ).

\section{Small molecule treatment and siRNA transfection}

Cells were plated in 24-well plates (for PCR or reporter assay) or 6-well plates (for western blot or wound-healing assay) 24 hours before treatment. Cells were transfected with $100 \mathrm{nmol} / \mathrm{L}$ of siRNA using Lipofectamine RNAiMAX (Invitrogen, Carlsbad, CA) according to the manufacturer's protocol. After transfection for 48 hours, cells were harvested for further 
analysis. Small molecule inhibitors CAY10561, FR180204 and Trametinib were dissolved in DMSO. Cells treated with small molecule inhibitors at a series of dosages were grown for 24 hours before being harvested.

\section{RNA isolation, cDNA synthesis and quantitative real-time RT-PCR}

Total RNA was extracted from cells using the RNeasy Mini kit (Qiagen, Valencia, CA). The cDNA was transcribed from $500 \mathrm{ng}$ of total RNA using iScript cDNA Synthesis Kits (Bio-Rad, Hercules, CA), according to the manufacturer's protocol. The cDNA was used as the template for real-time PCR detection using TaqMan Technology on an Applied Biosystems 7000 sequence detection system (Applied Biosystems, Foster City, CA). Expression of BIRC5, CTGF, Gli2 genes and endogenous control gene b-glucuronidase (GUSB) were detected using the primer and probe sequences commercially available (Applied Biosystems) and analyzed using Relative Quantification Software (Applied Biosystems).

\section{Luciferase reporter assay}

The $8 \times$ GTIIC-luciferase plasmid (Addgene, Cambridge, MA) and Renilla luciferase pRL-TK plasmid (Promega, Madison, WI) were co-transfected into cell lines. The transfection reagents were Lipofectamine RNAiMAX or Lipofectamine 2000 (Invitrogen, Carlsbad, CA), depending on treatment with siRNA or small molecule inhibitors. After 48 hours, cells were harvested and transferred into a 96-well plate for analysis by using the Dual-Luciferase Reporter Assay Kit (Promega, Madison, WI). Detection of luminescent signaling was performed on a GloMax-96 Microplate Luminometer (Promega, Madison, WI) according to the manufacturer's instructions.

\section{Western blot analysis}

Total protein was extracted from cell lines using M-PER Mammalian Protein Extraction Reagent (Thermo) supplied with Complete Protease Inhibitor Cocktails (Roche, Lewes, UK), according to manufacturers' protocols. The protein concentrations were measured with the Pierce BCA Protein Assay Kit (Thermo). A total of $10 \mu \mathrm{g}$ of proteins were run on $4 \sim 20 \%$ gradient SDS-polyacrylamide gels (Bio-Rad Laboratories, Inc., Hercules, CA) and transferred to Immobilon-P nitrocellulose membranes (Millipore, Bellerica, MA). The membranes were blocked in 5\% nonfat milk and then probed with the primary antibodies overnight at $4^{\circ} \mathrm{C}$. The membranes were incubated with appropriate secondary antibodies, followed by detection using an ECL blotting analysis system (Amersham Pharmacia Biotech, Piscataway, NJ).

\section{Protein degradation assay}

The ERK1/2 siRNA and control siRNA in a concentration of $100 \mathrm{nM}$ were transfected into the NSCLC cells. After 48 hours of transfection, the cells were treated with $100 \mu \mathrm{g} / \mathrm{ml}$ cycloheximide (Sigma), the inhibitor of protein synthesis, and harvested at the time points of 0 , 1, 2, 4, 6 and 8 hours. Total proteins were extracted and expression of YAP was analyzed by western blot.

\section{Cell viability assay}

Cells were cultured in a 96-well plate and treated with different doses of ERK inhibitors (CA10561: 0, 0.01, 0.03, 0.1, 0.3, 1, 3, 10, 30, $100 \mu \mathrm{M}$; FR180204: 0, 0.3, 1, 3, 10, $30,100,300,1000,3000 \mu \mathrm{M})$. After 48 hours of incubation, cells were lysed and luminescent signaling was generated by a CellTiter-Glo Luminescent Cell Viability Assay reagent (Promega). Luminescent signaling was measured on the GloMax-96 Microplate Luminometer. Proportional cell viability was analyzed with GraphPad Prism6 software (GraphPad Software, Inc., La Jolla, CA), which was used to calculate dose-response curves and IC50.

\section{Wound-healing assay}

Sub-confluent cultures of cell lines were transfected with ERK1, ERK2, ERK1/2, YAP or control siRNAs. The plates were scratched by a $200 \mu$ pipette tip at 48 hours after transfection. The fresh medium was replaced and cells were grown continuously. Phase contrast images were taken with a Primo Vert microscope (ZEISS, Gottingen, Germany) at the time of the scratch $(0 \mathrm{~h})$ and every 6 hours after. Scratch areas were quantified using ImageJ software. Wound-closure rates were calculated as percentages of the initial distance $(0 \mathrm{~h})$, and normalized using the data from control siRNA.

\section{Transwell invasion assay}

The transwell invasion assay was performed in a 6-well plate transwell system (Corning Incorporated, USA). The transwell inserts were coated with $100 \mu \mathrm{l}$ matrigel and incubated at $37^{\circ} \mathrm{C}$ for 2 hours. H1975 cells were transfected with $100 \mathrm{nM}$ control siRNA, ERK2 siRNA or YAP siRNA for 48 hours. After siRNA treatment, cells were harvested and resuspended in serumfree medium. The cells were seeded on the upper chamber of the transwell, and the lower chamber was infused with $2 \mathrm{ml}$ complete growth medium $(10 \% \mathrm{FBS})$. The transwell was incubated at $37^{\circ} \mathrm{C}$ for 20 hours, and then the gel and cells in the upper chamber were wiped out. After methanol fixation, the membrane was stained by hematoxylin for 40 seconds. Phase contrast images were taken and the cells on the lower side of the membrane were counted in six random visual fields under a $20 \times$ objective lens. 


\section{Tissue samples and immunohistochemistry (IHC)}

Fresh lung tumor tissues were obtained from patients who were undergoing surgical resection of the primary tumor. All human tissue samples were obtained and analyzed in accordance with procedures approved by the institutional review board of the University of California, San Francisco (IRB H8714-22 942-01). The tissue microarray sections were immunostained as previously described [55]. The following scoring system was used: -, no stain; + , weak staining $(10 \%$ or above stained cellularity considered as positive); ++ , moderate staining ( $30 \%$ or above stained cellularity considered as positive); +++ , strong staining ( $50 \%$ or above stained cellularity considered as positive). All scoring was done under low power objective lens $(20 \times)$ with a Zeiss Axioscop 2 microscope (Carl Zeiss Inc, Germany). Images were taken under $20 \times$ or $40 \times$ objective lens.

\section{Statistical analysis}

Data are expressed as mean \pm standard deviation (SD) from three independent experiments. All statistical analyses were performed using the SPSS 17.0 for Windows software system (SPSS Inc, Chicago, IL). Oneway ANOVA followed by Scheffe multiple comparisons were used to compare the differences among multiple groups. A significant difference was considered when the $P$ value from a two-tailed test was $<0.05$.

\section{ACKNOWLEDGMENTS}

This study was supported by the National Institutes of Health (NIH; Grant No. R01 CA140654, to LY). We are grateful for support from the Kazan, McClain, Abrams, Fernandez, Lyons, Greenwood, Harley and Oberman Foundation, Inc.; the Estate of Robert Griffiths; the Jeffrey and Karen Peterson Family Foundation; Paul and Michelle Zygielbaum; the Estate of Norman Mancini; and the Barbara Isackson Lung Cancer Research Fund. We thank Pamela Derish in the UCSF Department of Surgery for editorial assistance with the manuscript.

\section{Conflict of interests statement}

All authors have no conflicts of interest.

\section{REFERENCES}

1. Molina JR, Adjei AA. The Ras/Raf/MAPK pathway. J Thorac Oncol. 2006; 1:7-9.

2. Zimmer S, Kahl P, Buhl TM, Steiner S, Wardelmann E, Merkelbach-Bruse S, Buettner R, Heukamp LC. Epidermal growth factor receptor mutations in non-small cell lung cancer influence downstream Akt, MAPK, and Stat3 signaling. J Cancer Res Clin Oncol. 2009; 135:723-730.
3. Kitano H, Chung JY, Ylaya K, Conway C, Takikita M, Fukuoka J, Doki Y, Hanaoka J, Hewitt SM. Profiling of Phospho-AKT, Phospho-mTOR, Phospho-MAPK and EGFR in Non-small Cell Lung Cancer. J Histochem Cytochem 2014, 62(5):335-346

4. Ramos JW. The regulation of extracellular signal-regulated kinase (ERK) in mammalian cells. Int J Biochem Cell Biol. 2008; 40:2707-2719.

5. Li C, Fan S, Owonikoko TK, Khuri FR, Sun SY, Li R. Oncogenic role of EAPII in lung cancer development and its activation of the MAPK-ERK pathway. Oncogene. 2011; 30:3802-3812.

6. Lu Z, Ding L, Hong H, Hoggard J, Lu Q, Chen YH. Claudin-7 inhibits human lung cancer cell migration and invasion through ERK/MAPK signaling pathway. Exp Cell Res. 2011; 317:1935-1946.

7. Neuzillet C, Hammel P, Tijeras-Raballand A, Couvelard A, Raymond E. Targeting the Ras-ERK pathway in pancreatic adenocarcinoma. Cancer Metastasis Rev. 2013; 32:147-162.

8. Chappell WH, Steelman LS, Long JM, Kempf RC, Abrams SL, Franklin RA, Basecke J, Stivala F, Donia M, Fagone P, Malaponte G, Mazzarino MC, Nicoletti F, et al. Ras/Raf/MEK/ERK and PI3K/PTEN/Akt/mTOR inhibitors: rationale and importance to inhibiting these pathways in human health. Oncotarget. 2011; 2:135-164.

9. Spirli C, Morell CM, Locatelli L, Okolicsanyi S, Ferrero C, Kim AK, Fabris L, Fiorotto R, Strazzabosco M. Cyclic AMP/PKA-dependent paradoxical activation of Raf/MEK/ ERK signaling in polycystin-2 defective mice treated with sorafenib. Hepatology. 2012; 56:2363-2374.

10. Hatzivassiliou G, Liu B, O'Brien C, Spoerke JM, Hoeflich KP, Haverty PM, Soriano R, Forrest WF, Heldens S, Chen H, Toy K, Ha C, Zhou W, et al. ERK inhibition overcomes acquired resistance to MEK inhibitors. Mol Cancer Ther. 2012; 11:1143-1154.

11. Morris EJ, Jha S, Restaino CR, Dayananth P, Zhu H, Cooper A, Carr D, Deng Y, Jin W, Black S, Long B, Liu J, Dinunzio E, et al. Discovery of a novel ERK inhibitor with activity in models of acquired resistance to BRAF and MEK inhibitors. Cancer Discov. 2013; 3:742-750.

12. Carlino MS, Todd JR, Gowrishankar K, Mijatov B, Pupo GM, Fung C, Snoyman S, Hersey P, Long GV, Kefford RF, Rizos H. Differential activity of MEK and ERK inhibitors in BRAF inhibitor resistant melanoma. Mol Oncol. 2014; 8:544-554.

13. Wang Y, Dong Q, Zhang Q, Li Z, Wang E, Qiu X. Overexpression of yes-associated protein contributes to progression and poor prognosis of non-small-cell lung cancer. Cancer Sci. 2010; 101:1279-1285.

14. Zhou Z, Hao Y, Liu N, Raptis L, Tsao MS, Yang X. TAZ is a novel oncogene in non-small cell lung cancer. Oncogene. 2011; 30:2181-2186. 
15. Rauch TA, Wang Z, Wu X, Kernstine KH, Riggs AD, Pfeifer GP. DNA methylation biomarkers for lung cancer. Tumour Biol. 2012; 33:287-296.

16. Zhao B, Li L, Tumaneng K, Wang CY, Guan KL. A coordinated phosphorylation by Lats and CK1 regulates YAP stability through SCF(beta-TRCP). Genes Dev. 2010; 24:72-85.

17. Wang K, Degerny C, Xu M, Yang XJ. YAP, TAZ, and Yorkie: a conserved family of signal-responsive transcriptional coregulators in animal development and human disease. Biochem Cell Biol. 2009; 87:77-91.

18. Lian I, Kim J, Okazawa H, Zhao J, Zhao B, Yu J, Chinnaiyan A, Israel MA, Goldstein LS, Abujarour R, Ding S, Guan KL. The role of YAP transcription coactivator in regulating stem cell self-renewal and differentiation. Genes Dev. 2010; 24:1106-1118.

19. Yu FX, Zhao B, Panupinthu N, Jewell JL, Lian I, Wang LH, Zhao J, Yuan H, Tumaneng K, Li H, Fu XD, Mills GB, Guan KL. Regulation of the Hippo-YAP pathway by G-protein-coupled receptor signaling. Cell. 2012; 150:780-791.

20. Guo X, Zhao B. Integration of mechanical and chemical signals by YAP and TAZ transcription coactivators. Cell Biosci. 2013; 3:33.

21. Zender L, Spector MS, Xue W, Flemming P, CordonCardo C, Silke J, Fan ST, Luk JM, Wigler M, Hannon GJ, $\mathrm{Mu} \mathrm{D}$, Lucito R, Powers S, et al. Identification and validation of oncogenes in liver cancer using an integrative oncogenomic approach. Cell. 2006; 125:1253-1267.

22. Steinhardt AA, Gayyed MF, Klein AP, Dong J, Maitra A, Pan D, Montgomery EA, Anders RA. Expression of Yesassociated protein in common solid tumors. Hum Pathol. 2008; 39:1582-1589.

23. Harvey KF, Zhang X, Thomas DM. The Hippo pathway and human cancer. Nat Rev Cancer. 2013; 13:246-257.

24. Kim D, Rath O, Kolch W, Cho KH. A hidden oncogenic positive feedback loop caused by crosstalk between Wnt and ERK pathways. Oncogene. 2007; 26:4571-4579.

25. Won JK, Yang HW, Shin SY, Lee JH, Heo WD, Cho KH. The crossregulation between ERK and PI3K signaling pathways determines the tumoricidal efficacy of MEK inhibitor. J Mol Cell Biol. 2012; 4:153-163.

26. Niederst MJ, Engelman JA. Bypass mechanisms of resistance to receptor tyrosine kinase inhibition in lung cancer. Sci Signal. 2013; 6re6.

27. Aronov AM, Baker C, Bemis GW, Cao J, Chen G, Ford PJ, Germann UA, Green J, Hale MR, Jacobs M, Janetka JW, Maltais F, Martinez-Botella G, et al. Flipped out: structureguided design of selective pyrazolylpyrrole ERK inhibitors. J Med Chem. 2007; 50:1280-1287.

28. Ohori M, Takeuchi M, Maruki R, Nakajima H, Miyake H. FR180204, a novel and selective inhibitor of extracellular signal-regulated kinase, ameliorates collagen-induced arthritis in mice. Naunyn Schmiedebergs Arch Pharmacol. 2007; 374:311-316.

29. Yamaguchi T, Kakefuda R, Tajima N, Sowa Y, Sakai T. Antitumor activities of JTP-74057 (GSK1120212), a novel MEK1/2 inhibitor, on colorectal cancer cell lines in vitro and in vivo. Int J Oncol. 2011; 39:23-31.

30. Li L, Wang J, Zhang Y, Ma L, Weng W, Qiao Y, Xiao W, Wang H, Yu W, Pan Q, He Y, Sun F. MEK1 promotes YAP and their interaction is critical for tumorigenesis in liver cancer. FEBS Lett. 2013; 587:3921-3927.

31. Zhao B, Tumaneng K, Guan KL. The Hippo pathway in organ size control, tissue regeneration and stem cell selfrenewal. Nat Cell Biol. 2011; 13:877-883.

32. Ramos A, Camargo FD. The Hippo signaling pathway and stem cell biology. Trends Cell Biol. 2012; 22:339-346.

33. Hiemer SE, Varelas X. Stem cell regulation by the Hippo pathway. Biochim Biophys Acta. 2013; 1830:2323-2334.

34. Lai D, Yang X. BMP4 is a novel transcriptional target and mediator of mammary cell migration downstream of the Hippo pathway component TAZ. Cell Signal. 2013; 25:1720-1728.

35. Artemenko Y, Batsios P, Borleis J, Gagnon Z, Lee J, Rohlfs M, Sanseau D, Willard SS, Schleicher M, Devreotes PN. Tumor suppressor Hippo/MST1 kinase mediates chemotaxis by regulating spreading and adhesion. Proc Natl Acad Sci U S A. 2012; 109:13632-13637.

36. Artemenko Y, Devreotes PN. Hippo on the move: tumor suppressor regulates adhesion and migration. Cell Cycle. 2013; 12:535-536.

37. Boguslawski G, McGlynn PW, Harvey KA, Kovala AT. SU1498, an inhibitor of vascular endothelial growth factor receptor 2, causes accumulation of phosphorylated ERK kinases and inhibits their activity in vivo and in vitro. J Biol Chem. 2004; 279:5716-5724.

38. McCubrey JA, Steelman LS, Chappell WH, Abrams SL, Wong EW, Chang F, Lehmann B, Terrian DM, Milella M, Tafuri A, Stivala F, Libra M, Basecke J, et al. Roles of the Raf/MEK/ERK pathway in cell growth, malignant transformation and drug resistance. Biochim Biophys Acta. 2007; 1773:1263-1284.

39. Aronov AM, Tang Q, Martinez-Botella G, Bemis GW, Cao J, Chen G, Ewing NP, Ford PJ, Germann UA, Green J, Hale MR, Jacobs M, Janetka JW, et al. Structure-guided design of potent and selective pyrimidylpyrrole inhibitors of extracellular signal-regulated kinase (ERK) using conformational control. J Med Chem. 2009; 52:6362-6368.

40. Samatar AA, Poulikakos PI. Targeting RAS-ERK signalling in cancer: promises and challenges. Nat Rev Drug Discov. 2014; 13:928-942.

41. Johnson R, Halder G. The two faces of Hippo: targeting the Hippo pathway for regenerative medicine and cancer treatment. Nat Rev Drug Discov. 2014; 13:63-79. 
42. Finger EC, Cheng CF, Williams TR, Rankin EB, Bedogni B, Tachiki L, Spong S, Giaccia AJ, Powell MB. CTGF is a therapeutic target for metastatic melanoma. Oncogene. 2014; 33:1093-1100.

43. Chu CY, Chang CC, Prakash E, Kuo ML. Connective tissue growth factor (CTGF) and cancer progression. J Biomed Sci. 2008; 15:675-685.

44. Yamamoto H, Ngan CY, Monden M. Cancer cells survive with survivin. Cancer Sci. 2008; 99:1709-1714.

45. Pantazi E, Gemenetzidis E, Trigiante G, Warnes G, Shan L, Mao X, Ikram M, Teh MT, Lu YJ, Philpott MP. GLI2 induces genomic instability in human keratinocytes by inhibiting apoptosis. Cell Death Dis. 2014; 5:e1028.

46. Sawada A, Kiyonari H, Ukita K, Nishioka N, Imuta Y, Sasaki H. Redundant roles of Tead1 and Tead 2 in notochord development and the regulation of cell proliferation and survival. Mol Cell Biol. 2008; 28:3177-3189.

47. Lin XY, Zhang XP, Wu JH, Qiu XS, Wang EH. Expression of LATS1 contributes to good prognosis and can negatively regulate YAP oncoprotein in non-small-cell lung cancer. Tumour Biol. 2014; 35:6435-6443.

48. Xu CM, Liu WW, Liu CJ, Wen C, Lu HF, Wan FS. Mst1 overexpression inhibited the growth of human non-small cell lung cancer in vitro and in vivo. Cancer Gene Ther. $2013 ; 20: 453-460$.
49. Gumbiner BM, Kim NG. The Hippo-YAP signaling pathway and contact inhibition of growth. J Cell Sci 2014, (Pt 4). 127:709-717.

50. Fan R, Kim NG, Gumbiner BM. Regulation of Hippo pathway by mitogenic growth factors via phosphoinositide 3-kinase and phosphoinositide-dependent kinase-1. Proc Natl Acad Sci U S A. 2013; 110:2569-2574.

51. Reddy BV, Irvine KD. Regulation of Hippo signaling by EGFR-MAPK signaling through Ajuba family proteins. Dev Cell. 2013; 24:459-471.

52. Yang S, Ji M, Zhang L, Chen Y, Wennmann DO, Kremerskothen J, Dong J. Phosphorylation of KIBRA by the extracellular signal-regulated kinase (ERK)-ribosomal S6 kinase (RSK) cascade modulates cell proliferation and migration. Cell Signal. 2014; 26:343-351.

53. Romano D, Nguyen LK, Matallanas D, Halasz M, Doherty C, Kholodenko BN, Kolch W. Protein interaction switches coordinate Raf-1 and MST2/Hippo signalling. Nat Cell Biol. 2014; 16:673-684.

54. Roskoski R Jr. ERK1/2 MAP kinases: structure, function, and regulation. Pharmacol Res. 2012; 66:105-143.

55. Li T, Li H, Wang Y, Harvard C, Tan JL, Au A, Xu Z, Jablons DM, You L. The expression of CXCR4, CXCL12 and CXCR7 in malignant pleural mesothelioma. J Pathol. 2011; 223:519-530. 